\title{
DETERMINAÇÃO ESPECTROFOTOMÉTRICA SIMULTÂNEA DE PARACETAMOL E IBUPROFENO EM FORMULAÇÕES FARMACÊUTICAS USANDO CALIBRAÇÃO MULTIVARIADA
}

\author{
Marcelo M. Sena*, Camilla B. Freitas, Lucas C. Silva, Caridad Noda Pérez e Ydilla O. de Paula \\ Unidade Universitária de Ciências Exatas e Tecnológicas, Universidade Estadual de Goiás, BR 153, km 98, \\ 75000-000 Anápolis - GO, Brasil
}

Recebido em 18/1/06; aceito em 7/4/06; publicado na web em 11/8/06

\begin{abstract}
SIMULTANEOUS SPECTROPHOTOMETRIC DETERMINATION OF PARACETAMOL AND IBUPROFEN IN PHARMACEUTICAL FORMULATIONS BY MULTIVARIATE CALIBRATION. A simple method was proposed for determination of paracetamol and ibuprofen in tablets, based on UV measurements and partial least squares. The procedure was performed at $\mathrm{pH}$ 10.5, in the concentration ranges 3.00-15.00 $\mathrm{g} \mathrm{ml}^{-1}$ (paracetamol) and 2.40-12.00 $\mathrm{g} \mathrm{ml}^{-1}$ (ibuprofen). The model was able to predict paracetamol and ibuprofen in synthetic mixtures with root mean squares errors of prediction of 0.12 and $0.17 \mu \mathrm{g} \mathrm{ml}^{-1}$, respectively. Figures of merit (sensitivity, limit of detection and precision) were also estimated. The results achieved for the determination of these drugs in pharmaceutical formulations were in agreement with label claims and verified by HPLC.
\end{abstract}

Keywords: PLS; quality control; acetaminophen.

\section{INTRODUÇÃO}

Nos últimos anos, a combinação entre métodos quimiométricos e técnicas de espectroscopia molecular apresentou grandes contribuições para a química analítica, tornando possível, por ex., a determinação de espécies sem resolução de sinal analítico. Uma das aplicações para a qual essa combinação demonstrou importância foi a determinação direta de fármacos. Embora a Farmacopéia americana "USP, United States Pharmacopoeia"1, não recomende metodologias multivariadas, uma revisão recente da literatura mostrou um aumento do número de publicações propondo o uso de métodos quimiométricos ${ }^{2}$. Atualmente, a maioria das metodologias propostas para determinação de fármacos é baseada em técnicas cromatográficas e determinações univariadas. Apesar dessas metodologias estarem bem estabelecidas e serem reconhecidas pelos organismos reguladores, elas podem apresentar desvantagens em certas situações, tais como custo e tempo de análise relativamente altos. Desta forma, a partir da última década, o emprego de espectroscopia molecular e calibração multivariada passou a representar uma alternativa promissora na determinação direta de fármacos e o desenvolvimento deste tipo de método tornou-se importante, tendo como objetivo sua futura aceitação pelas agências reguladoras. Como consequência disso, tornou-se necessário também o estabelecimento de figuras de mérito para metodologias multivariadas, requisito para sua validação analítica ${ }^{3}$.

Em situações nas quais a determinação de um analito é dificultada ou impedida devido à presença de interferentes, o uso de calibração multivariada permite a modelagem desses interferentes junto com o analito de interesse. A idéia central consiste em substituir a separação física das espécies pela separação quimiométrica de seus sinais. O método de calibração multivariada mais conhecido é o dos mínimos quadrados parciais "PLS, Partial Least Squares" 4,5 , usado na construção de modelos de regressão baseados em uma decomposição em variáveis latentes, a qual relaciona um bloco de variáveis independente, $\mathrm{x}$ (espectros), com um bloco de variáveis dependentes, y (concentrações ou outras propriedades). A

*e-mail: marcsen@ueg.br estratégia que combina PLS e dados obtidos por espectrofotometria de absorção no ultravioleta (UV) tem permitido a determinação direta e simultânea de diversos princípios ativos em uma mesma formulação farmacêutica, tais como aspirina, cafeína e codeína ${ }^{6}$; amilorida e hidroclorotiazida ${ }^{7}$; aspirina e ácido ascórbico ${ }^{8}$; aspirina, paracetamol e cafeína ${ }^{9}$; pseudoefedrina e ibuprofeno ${ }^{10}$; diclofenaco, tiamina e piridoxina ${ }^{11}$. As vantagens potenciais desta estratégia são rapidez, redução de uso de reagentes, baixo custo e simplificação no preparo da amostra.

O paracetamol (PRC) e o ibuprofeno (IBF), cujas estruturas moleculares são apresentadas na Figura 1, estão entre os fármacos mais consumidos no mundo. O PRC ${ }^{12}$, também conhecido como acetaminofeno, possui efeitos analgésico e antipirético similares aos da aspirina, apresentando a vantagem de não irritar a mucosa gastrointestinal. $\mathrm{O} \mathrm{IBF}^{13}$ é uma droga não-esteroidal anti-inflamatória que também possui efeitos analgésico e antipirético. Algumas formulações farmacêuticas que combinam estes dois princípios ativos são usadas no tratamento de dores moderadas de origem reumática. Vários métodos já foram empregados na determinação individual de cada um destes dois fármacos em comprimidos, tais como espectrofotométricos ${ }^{14-17}$, cromatográficos ${ }^{18,19}$ e potenciométricos ${ }^{20}$ para o PRC e espectrofotométricos ${ }^{21,22}$, cromatográficos ${ }^{23-26} \mathrm{e}$ eletroforéticos ${ }^{27,28}$ para o IBF. Os métodos oficiais (USP) para determinação das duas espécies são baseados em cromatografia líquida de alta eficiência (CLAE) com deteção no UV ${ }^{1}$. No entanto, a determinação simultânea de PRC e IBF é relatada em poucos artigos. Em um deles, essa determinação é feita através de ressonância magnética nuclear de próton ${ }^{29}$, uma técnica cara e relativamente pouco acessível à maioria dos laboratórios de análise, enquanto em outro, utilizou-se espectrofotometria e regressão linear múltipla ${ }^{30}$,<smiles>CC(=O)Nc1ccc(O)cc1</smiles>

Figura 1. Fórmulas estruturais do PRC e do IBF 
a qual está limitada ao uso de apenas alguns poucos comprimentos de onda. Para nenhum destes dois métodos foram estimadas figuras de mérito. O fato de PRC e IBF apresentarem espectros de absorção sobrepostos no UV ${ }^{12,13}$ impede que sejam realizadas determinações espectrofotométricas diretas e simultâneas. Daí, o interesse deste trabalho em propor um método direto, simples e de baixo custo para essa determinação, usando o PLS para construir um modelo de calibração a partir dos espectros de um número relativamente reduzido (nove) de soluções de misturas de padrões. Um outro objetivo deste trabalho foi estimar figuras de mérito para o método proposto. Os resultados das análises de amostras comerciais foram ainda verificados por uma técnica analítica independente, cromatografia líquida de alta eficiência (CLAE).

\section{PARTE EXPERIMENTAL}

\section{Reagentes e soluções}

Os reagentes utilizados foram de pureza analítica ou grau cromatográfico: PRC (Synth), IBF (Aldrich), hidróxido de amônio (Synth), cloreto de amônio (Vetec), metanol (Tedia), acetonitrila (JTBaker) e ácido acético (CRQ). Duas soluções estoque, PRC 1000,0 e IBF 800,0 $\mu \mathrm{g} \mathrm{mL}^{-1}$, foram preparadas em metanol, em balões de $100 \mathrm{~mL}$. A dissolução foi feita em metanol, por causa da baixa solubilidade do IBF em água ${ }^{13}$. Cinco soluções intermediárias de cada analito foram preparadas por diluição em água da respectiva solução estoque, nas faixas de 40 a $200 \mu \mathrm{g} \mathrm{mL}^{-1}$ para o PRC e de 32 a $160 \mu \mathrm{g} \mathrm{mL}^{-1}$ para o IBF. Essas soluções foram guardadas na geladeira $\left(4{ }^{\circ} \mathrm{C}\right)$ em frascos protegidos da luz e mostraram-se estáveis por pelo menos 2 meses. Uma solução tampão de $\mathrm{pH}$ 10,5 foi preparada a partir de $\mathrm{NH}_{4} \mathrm{Cl} / \mathrm{NH}_{4} \mathrm{OH}$. Toda a água utilizada foi previamente deionizada (Milli-Q).

\section{Instrumentação e tratamento dos dados}

Um espectrofotômetro UV/visível de arranjo dispersivo Cary 50 foi usado para aquisição dos dados, juntamente com o Cary WinUV Software, versão 3.00. Os dados foram tratados no programa MATLAB ${ }^{T M}$, versão 6.1 (The MathWorks, Natick, EUA), usando o pacote PLS Toolbox, versão 2.0 (Eigenvector Technologies, Manson, EUA). Um banho ultra-sônico (Unique) foi utilizado na dissolução das amostras.

\section{Procedimentos}

\section{Conjunto de calibração e misturas sintéticas}

As soluções do conjunto de calibração foram preparadas de acordo com um planejamento experimental $3^{2}$ (dois fatores e três níveis, Tabela 1). As misturas sintéticas usadas como conjunto de validação foram preparadas de acordo com um planejamento $3^{2}$ similar ao da Tabela 1. Para este novo conjunto, os níveis superiores foram $12,00 \mu \mathrm{g} \mathrm{mL}^{-1}$ para PRC e 9,60 $\mathrm{g} \mathrm{mL}^{-1}$ para IBF, os níveis inferiores foram $6,00 \mu \mathrm{g} \mathrm{mL}^{-1}$ para PRC e $4,80 \mu \mathrm{g} \mathrm{mL}^{-1}$ para IBF e os níveis intermediários foram idênticos aos do conjunto de calibração. Três pontos do conjunto de validação, os dois extremos $(++$ e --$)$ e o central $(00)$, foram determinados em triplicata, visando a estimativa da precisão média do método. Desta forma, 24 soluções de trabalho (calibração e validação) foram preparadas diretamente na cubeta $(1,00 \mathrm{~cm}$ de caminho ótico) pela adição de $150,0 \mu \mathrm{L}$ da respectiva solução intermediária de cada analito e 2700 $\mu \mathrm{L}$ da solução tampão. Os espectros foram coletados na faixa de 220 a $320 \mathrm{~nm}$ (passo $1 \mathrm{~nm}$ ). A ordem em que as soluções foram analisadas foi sorteada, de modo a garantir a aleatoridade das me-
Tabela 1. Planejamento experimental $3^{2}$ para o conjunto de calibração usado na determinação simultânea de PRC e IBF ${ }^{a}$

\begin{tabular}{llllllllll}
\hline $\begin{array}{l}\text { Solução / } \\
\text { Analito }\end{array}$ & $\mathbf{1}$ & $\mathbf{2}$ & $\mathbf{3}$ & $\mathbf{4}$ & $\mathbf{5}$ & $\mathbf{6}$ & $\mathbf{7}$ & $\mathbf{8}$ & $\mathbf{9}$ \\
\hline $\mathrm{PRC}$ & + & + & + & 0 & 0 & 0 & - & - & - \\
$\mathrm{IBF}$ & + & 0 & - & + & 0 & - & + & 0 & - \\
\hline
\end{tabular}

${ }^{a}$ Nível (+): PRC 15,00 $\mu \mathrm{g} \mathrm{mL}^{-1}$ e IBF 12,00 $\mu \mathrm{g} \mathrm{mL}^{-1}$; Nível (0): PRC 9,00 $\mu \mathrm{g} \mathrm{mL}^{-1}$ e IBF 7,20 $\mu \mathrm{g} \mathrm{mL}^{-1}$; Nível (-): PRC 3,00 $\mu \mathrm{g} \mathrm{mL}^{-1}$ e IBF $2,40 \mu \mathrm{g} \mathrm{mL}^{-1}$

$\operatorname{didas}^{31}$. Uma solução preparada da mesma maneira que as soluções de trabalho, mas não contendo nenhum dos analitos, foi usada como branco. Espectros do branco foram medidos quinze vezes em seqüência, visando estimar o ruído instrumental para o cálculo de figuras de mérito. Espectros de soluções puras de PRC e IBF também foram registrados.

\section{Determinação das amostras comerciais}

Duas amostras de formulações farmacêuticas comerciais disponíveis no mercado nacional foram compradas em uma farmácia local. As duas amostras apresentaram a mesma composição, especificada pelos fabricantes: $200 \mathrm{mg}$ de IBF e $300 \mathrm{mg}$ de PRC. A amostra 1 apresentou a seguinte composição de excipientes: estearato de magnésio, amido de milho, Explotab, gelatina em pó, Microcel, talco e corante vermelho. A amostra 2 não especificou a composição de excipientes. Dez comprimidos de cada formulação foram pesados individualmente para se obter massas médias representativas. Os comprimidos foram finamente triturados em um almofariz de ágata e depois misturados. Massas correspondentes a exatamente $50,0 \%$ do valor médio de um comprimido para cada amostra foram dissolvidas em $100 \mathrm{~mL}$ de metanol, com o auxílio de um banho ultrassônico (10 min). Na seqüência, foram preparadas duas soluções intermediárias pela diluição de $10,00 \mathrm{~mL}$ da solução de cada amostra em água, em balões de 100,0 mL. Alíquotas de 150,0 $\mu \mathrm{L}$ de cada solução intermediária foram misturadas diretamente na cubeta com 150,0 $\mu \mathrm{L}$ de água e $2700 \mu \mathrm{L}$ da solução tampão de $\mathrm{pH} 10,5$. Os espectros destas soluções foram obtidos da mesma maneira descrita na seção anterior e as determinações foram feitas em triplicatas.

\section{Análise cromatográfica}

A verificação dos resultados das determinações de formulações farmacêuticas foi feita por CLAE, usando um método adaptado de Hussain et al. ${ }^{32}$. Foi utilizado um cromatógrafo líquido Varian, composto por uma bomba ternária modelo 240 , um detector no UV modelo 310 e um amostrador automático AutoSampler 410, comandados pelo programa Star, versão 6.0. Foi usada uma coluna analítica ChromSpher 5 C18, 250 x 4,6 mm (Varian). A fase móvel foi composta por acetonitrila/ácido acético 0,5\%, 65:35, v/v, em uma vazão de $1,0 \mathrm{~mL} \mathrm{~min}{ }^{-1}$. A detecção foi feita com mudança de comprimento de onda durante a corrida, sendo em $250 \mathrm{~nm}$ até 3,7 min (detecção do PRC) e em $223 \mathrm{~nm}$ após esse intervalo (detecção do IBF). O volume de injeção foi de $15 \mu \mathrm{L}$. Todas as injeções foram repetidas três vezes e as determinações foram feitas em triplicata.

\section{RESULTADOS E DISCUSSÃO}

\section{Espectros de absorção no UV de PRC e IBF}

A Figura 2 mostra os espectros de absorção no UV de soluções aquosas de PRC e de IBF, obtidos em pH 10,5 ([PRC] $=[\mathrm{IBF}]=$ $\left.12,00 \mu \mathrm{g} \mathrm{mL}^{-1}\right)$. O espectro do PRC possui uma banda larga com 
absorção máxima em 252 nm, enquanto o IBF apresenta um máximo em $222 \mathrm{~nm}$ e uma banda de baixa intensidade de absorção entre 245 e $280 \mathrm{~nm}$. Como pode ser observado, a sobreposição espectral, principalmente em relação ao IBF, impede o uso de calibração univariada em uma determinação direta e simultânea.

Os valores de $\mathrm{pK}_{\mathrm{a}}$ encontrados na literatura ${ }^{12,13,33,34}$ para o PRC e o IBF estão próximos de 9,5 e de 4,4, respectivamente. A forma ácida do $\mathrm{PRC}$, predominante abaixo de $\mathrm{pH}$ 9,5, apresenta um máximo de absorção em $243 \mathrm{~nm}$ (espectro obtido experimentalmente em $\mathrm{pH}$ 7), aumentando a sobreposição espectral com o IBF. Por esse motivo, escolheu-se trabalhar em $\mathrm{pH}$ 10,5 usando tampão $\mathrm{NH}_{4} \mathrm{Cl} /$ $\mathrm{NH}_{4} \mathrm{OH}$, que não absorve significativamente na região de interesse.

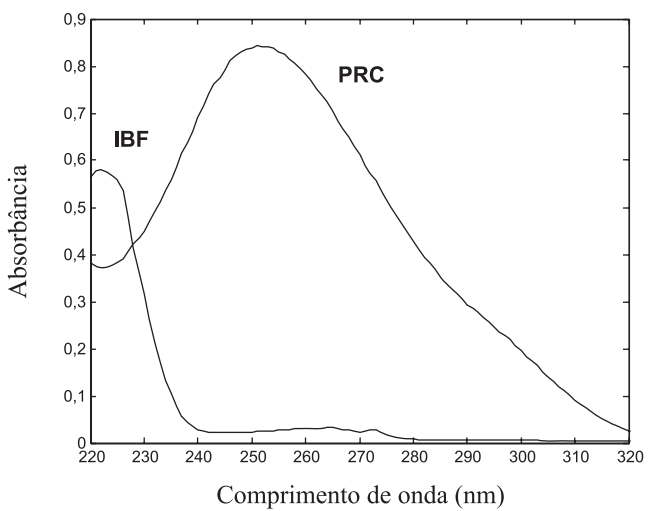

Figura 2. Espectros de absorção no UV do PRC e do IBF obtidos em pH 10,5. $[P R C]=[I B F]=12,00 \mu g m L^{-1}$

\section{Calibração multivariada usando PLS}

Uma das vantagens do método de determinação aqui proposto é o uso de um número reduzido de amostras de calibração, seguindo um planejamento experimental simples (Tabela 1). Um planejamento de calibração de três níveis poderia ser considerado insuficiente, pois normalmente quatro ou cinco níveis de concentração são recomendados para cada analito 5 . No entanto, essa escolha depende fortemente da natureza do sistema a ser modelado. Este planejamento reduzido certamente seria insuficiente para matrizes mais complexas, como, por ex., amostras de solo analisadas no infravermelho. Mas, ele pode ser considerado adequado para matrizes mais simples, tais como a analisada nesta aplicação, o que pode ser corroborado pelos bons resultados obtidos aqui e em outras aplicações encontradas na literatura ${ }^{6-11}$.

O melhor modelo PLS foi selecionado com três variáveis latentes através de um procedimento de validação cruzada "leave-oneout". Este modelo explicou 99,99\% da variância total do bloco X (variáveis independentes) e 99,95\% da variância do bloco Y (variáveis dependentes). A raiz quadrada dos erros médios quadrados de previsão ("RMSEP, Root Mean Square Error of Prediction") do conjunto de validação foi o parâmetro de exatidão inicialmente usado para avaliar o modelo. O RMSEP é calculado pela Equação 1, onde $\mathrm{y}_{\mathrm{r}}$ é o valor padrão (esperado) da concentração, $\hat{y}_{\mathrm{p}}$ é o valor previsto pelo modelo e n é o número de amostras previstas.

$\operatorname{RMSEP}=\sqrt{\frac{\sum\left(\mathrm{y}_{\mathrm{r}}-\hat{\mathrm{y}}_{\mathrm{p}}\right)^{2}}{\mathrm{n}}}$

Os valores de RMSEP obtidos foram $0,12 \mu \mathrm{g} \mathrm{mL}^{-1}$ para o PRC e $0,17 \mu \mathrm{g} \mathrm{mL}^{-1}$ para o IBF, correspondendo a uma recuperação que variou entre 98,4 e 103,7\% para o PRC e entre 96,9 e 104,8 \% para o IBF. As previsões individuais para as misturas sintéticas do conjunto de validação são mostradas na Tabela 2. Os resultados para a previsão do IBF foram um pouco menos exatos do que os relativos ao PRC, o que pode ser explicado pelo fato de o espectro do primeiro ser mais fortemente sobreposto.

Tabela 2. Determinação simultânea de PRC e IBF em quinze misturas sintéticas (conjunto de validação)

\begin{tabular}{rcccrr}
\hline \multicolumn{3}{c}{$\begin{array}{l}\text { Quantidade } \\
\text { adicionada }\left(\mu \mathrm{g} \mathrm{mL} \mathrm{mL}^{-1}\right)\end{array}$} & \multicolumn{2}{c}{ Quantidade } & \multicolumn{2}{c}{ Recuperação } \\
pRC & IBF & PRC & IBF & PRC & IBF \\
\hline 12,00 & 9,60 & 12,19 & 9,31 & 101,6 & 96,9 \\
12,00 & 9,60 & 11,88 & 9,76 & 99,0 & 101,7 \\
12,00 & 9,60 & 11,96 & 9,92 & 99,7 & 103,3 \\
12,00 & 7,20 & 12,05 & 7,28 & 100,4 & 101,1 \\
12,00 & 4,80 & 12,07 & 5,01 & 100,6 & 104,5 \\
9,00 & 9,60 & 9,08 & 9,71 & 100,9 & 101,1 \\
9,00 & 7,20 & 9,20 & 7,18 & 102,2 & 99,8 \\
9,00 & 7,20 & 8,93 & 7,16 & 99,3 & 99,4 \\
9,00 & 7,20 & 8,86 & 7,13 & 98,4 & 99,0 \\
9,00 & 4,80 & 8,92 & 5,03 & 99,1 & 104,8 \\
6,00 & 9,60 & 6,22 & 9,58 & 103,7 & 99,8 \\
6,00 & 7,20 & 5,97 & 7,37 & 99,4 & 102,3 \\
6,00 & 4,80 & 6,01 & 4,91 & 100,2 & 102,4 \\
6,00 & 4,80 & 6,05 & 4,92 & 100,8 & 102,4 \\
6,00 & 4,80 & 6,12 & 4,83 & 102,0 & 100,7 \\
\hline
\end{tabular}

\section{Figuras de mérito}

Os métodos analíticos baseados em calibração multivariada são relativamente recentes e sua aceitação e implementação ainda é restrita, principalmente em áreas como a farmacêutica e a médica, devido às exigências de validação pelas agências e organismos reguladores ${ }^{35,36}$. Essa validação pode ser acessada pela determinação de parâmetros que são conhecidos como figuras de mérito, tais como sensibilidade, precisão e limite de detecção (além da exatidão, já discutida na seção anterior). Na estimativa de figuras de mérito em calibração multivariada ${ }^{3}$, a parte do sinal que se relaciona unicamente ao analito de interesse é mais importante que o sinal total e é denominada sinal analítico líquido ou NAS "Net Analyte Signal". O NAS é definido como a parte do sinal analítico que é ortogonal ao sinal dos interferentes presentes na amostra ${ }^{37}$. O vetor NAS contém os valores para cada amostra e pode ser relacionado com o vetor dos coeficientes de regressão, b, em modelos de calibração inversa, tais como o PLS, através da seguinte Equação ${ }^{38}$ :

$\|\mathbf{N A S}\|=1 /\|\mathbf{b}\|$

onde o símbolo " $\mid$ ||" indica a norma euclideana de um vetor. Outras figuras de mérito podem ser calculadas como função do $\mathbf{N A S}{ }^{38}$. A sensibilidade (SEN), a fração da variação do sinal associada ao acréscimo de uma unidade de concentração, pode ser expressa pela Equação:

$\mathrm{SEN}=\|\mathbf{N A S}\|$

A sensibilidade analítica $(\gamma)$, normalmente não abordada em protocolos de validação, apresenta, de forma clara, a sensibilidade do método em função da unidade de concentração que é utilizada. Em analogia com a calibração univariada ${ }^{39}, \gamma$ é definida como a razão entre a SEN e o ruído instrumental $(\varepsilon)$, de acordo com a Equação 4 . O vetor $\boldsymbol{\varepsilon}$ contém o desvio padrão do sinal analítico em cada comprimento de onda e, neste trabalho, foi estimado a partir de quinze replicatas do branco. 
$\gamma=\mathrm{SEN} /\|\varepsilon\|$

O limite de detecção (LD), a menor quantidade da espécie de interesse que pode ser detectada sob as condições experimentais estabelecidas, é estimado através da Equação:

$\mathrm{LD}=3\|\varepsilon\| /\|\mathbf{N A S}\|$

A precisão expressa o grau de concordância entre os resultados de uma série de medidas feitas para uma mesma amostra homogênea, em condições determinadas. Neste trabalho, ela foi considerada no nível de repetibilidade, a partir de nove determinações (três níveis de concentrações e três replicatas) cobrindo a faixa útil de previsão do modelo (os dois níveis extremos mais o central). A precisão média foi estimada de acordo com a seguinte Equação:

precisão média $=\sqrt{\frac{\sum_{i}^{n} \sum_{j}^{m}\left(\hat{y}_{i j}-\hat{\bar{y}}_{i}\right)^{2}}{n(m-1)}}$

na qual $\hat{y}_{\mathrm{ij}}$ é o valor estimado para cada replicata, $\hat{\bar{y}}_{\mathrm{i}}$ é a média estimada para cada amostra, $n$ é o número de amostras replicadas e m é o número de replicatas por amostra.

A Tabela 3 mostra as figuras de mérito estimadas para as determinações do PRC e do IBF. Como o inverso da $\gamma$ fornece a diferença de concentração mínima que é discernível pelo método analítico, independente da técnica específica empregada, pode-se estimar que o método é capaz de distinguir diferenças de concentração de $0,01 \mu \mathrm{g} \mathrm{mL}^{-1}$ de PRC e de $0,03 \mu \mathrm{g} \mathrm{mL}^{-1}$ de IBF, na ausência de erro experimental. As estimativas de sensibilidade analítica e de precisão média obtidas atestaram a eficiência do modelo. Além da precisão média, os valores de desvio padrão relativo para as previsões de PRC e IBF nos três níveis do conjunto de validação replicados são apresentados na Tabela 4.

Tabela 3. Figuras de mérito para determinação simultânea de PRC e IBF, usando o modelo PLS

\begin{tabular}{lcc}
\hline Figuras de mérito & PRC & IBF \\
\hline $\mathrm{SEN}^{a}$ & 0,24 & 0,09 \\
$\mathrm{~g}\left(\mathrm{~mL} \mu \mathrm{g}^{-1}\right)$ & 92 & 33 \\
$\mathrm{LD}\left(\mu \mathrm{gL} \mathrm{m}^{-1}\right)$ & 0,03 & 0,09 \\
precisão média $\left(\mu \mathrm{g} \mathrm{mL}^{-1}\right)$ & 0,14 & 0,19 \\
\hline
\end{tabular}

${ }^{a}$ Valor expresso como a razão entre a derivada da absorbância e a unidade de concentração $\left(\mu \mathrm{g} \mathrm{mL}^{-1}\right)$

\section{Determinação de amostras comerciais e verificação por CLAE}

Duas formulações farmacêuticas disponíveis no mercado brasileiro foram analisadas. Os resultados das previsões são mostra-
Tabela 4. Valores de desvio padrão relativo para a previsão simultânea de PRC e IBF em triplicatas de três níveis do conjunto de validação

\begin{tabular}{lcc}
\hline Nível $^{a}$ & PRC & IBF \\
\hline superior & 1,3 & 3,3 \\
intermediário & 2,0 & 0,4 \\
inferior & 1,0 & 1,0 \\
\hline
\end{tabular}

${ }^{a}$ superior: PRC 12,00 $\mu \mathrm{g} \mathrm{mL}^{-1}$ e IBF 9,60 $\mu \mathrm{g} \mathrm{mL}^{-1}$; intermediário: PRC 9,00 $\mu \mathrm{g} \mathrm{mL}^{-1}$ e IBF 7,20 $\mu \mathrm{g} \mathrm{mL}^{-1}$; inferior: PRC 6,00 $\mu \mathrm{g} \mathrm{mL}^{-1} \mathrm{e}$ IBF $4,80 \mu \mathrm{g} \mathrm{mL}^{-1}$

dos na Tabela 5. As diferenças em relação aos valores especificados pelos fabricantes variaram entre 3,8 e $8,4 \%$. Estes resultados podem ser considerados em acordo com o especificado, levandose em conta o limite de tolerância de $\pm 10 \%$ estabelecido pela Farmacopéia americana para este tipo de fármaco ${ }^{1}$.

Para verificar esses resultados, as amostras comerciais foram analisadas por uma segunda metodologia, baseada em uma outra técnica analítica, CLAE. Assim como as determinações espectrofotométricas, as determinações por CLAE também foram feitas em triplicata, mas usando amostras preparadas de forma independente. Um teste t com quatro graus de liberdade foi realizado para mostrar que os resultados das duas metodologias não diferiram significativamente num nível de $95 \%$ de confiança $(t=1,02$ para o PRC e $\mathrm{t}=0,27$ para o IBF na amostra 1 e $\mathrm{t}=0,44$ para o PRC e $\mathrm{t}=$ 1,40 para o IBF na amostra 2 , todos bem abaixo do valor crítico de $2,78)$.

\section{CONCLUSÃO}

A combinação de PLS e espectrofotometria no UV mostrou-se uma alternativa viável e de relativo baixo custo para determinação simultânea de PRC e IBF, fornecendo previsões exatas e precisas para misturas sintéticas e amostras de formulações comerciais. Além disso, a estimativa de figuras de mérito, tais como sensibilidade e limite de detecção, e a verificação dos resultados das previsões de amostras comerciais usando outra técnica analítica, CLAE, atestaram a eficiência do método. Desta forma, o método proposto pode ser sugerido como uma possível alternativa para o controle de qualidade destes fármacos neste tipo de formulação farmacêutica. Esse método poderia ainda ser automatizado em um sistema de injeção em fluxo, o que levaria a outras vantagens, tais como maior rapidez e diminuição dos erros, devido à menor manipulação das amostras.

\section{AGRADECIMENTOS}

À FINEP pelas aquisições do espectrofotômetro UV/vis e do cromatógrafo, dentro do projeto CPDM. C. B. Freitas agradece ao PBIC-UEG pela concessão de uma bolsa de Iniciação Científica.

Tabela 5. Determinação simultânea de PRC e IBF em duas formulações farmacêuticas pelo método proposto (espectrofotométrico/ quimiométrico) e por CLAE

\begin{tabular}{lcccccc}
\hline Formulação & \multicolumn{2}{c}{ Valor especificado (mg) } & \multicolumn{2}{c}{ Método proposto (mg) $)^{a}$} & \multicolumn{2}{c}{ CLAE (mg) } \\
& PRC & IBF & PRC & IBF & PRC & IBF \\
\hline$\# 1$ & 300 & 200 & $279,0 \pm 4,2$ & $189,5 \pm 4,2$ & $270,1 \pm 14,6$ & $187,9 \pm 9,2$ \\
$\# 2$ & 300 & 200 & $274,8 \pm 3,5$ & $192,5 \pm 2,6$ & $276,8 \pm 7,1$ & $198,2 \pm 6,6$ \\
\hline
\end{tabular}

${ }^{a}$ Valores médios e desvios padrão de três determinações 


\section{REFERÊNCIAS}

1. The United States Pharmacopoeia; $25^{\mathrm{a}}$ rev., U.S.P. Convention: Rockville, 2002.

2. Gilpin, R. K.; Pachla, L. A.; Anal. Chem. 2005, 77, 3755.

3. Braga, J. W. B.; Poppi, R. J.; Quim. Nova 2004, 27, 1004.

4. Martens, H.; Naes, T.; Multivariate Calibration, Wiley: Chichester, 1989.

5. Brereton, R. G.; Analyst 2000, 125, 2125.

6. Bautista, R. D.; Jimenez, A. I.; Jimenez, F.; Arias, J. J.; Fresenius J. Anal. Chem. 1997, 357, 449.

7. Ferraro, M. C. F.; Castellano, P. M.; Kaufman, T. S.; J. Pharm. Biomed. Anal. 2002, 30, 1121.

8. Sena, M. M.; Fernandes, J. C. B.; Rover Jr, L.; Poppi, R. J.; Kubota, L. T.; Anal. Chim. Acta 2000, 409, 159.

9. Sena, M. M.; Poppi, R. J.; J. Pharm. Biomed. Anal. 2004, 34, 27.

10. Palabiyik, I. M.; Dinc, E.; Onur, F.; J. Pharm. Biomed. Anal. 2004, 34, 473.

11. Sena, M. M.; Chaudhry, Z. F.; Collins, C. H.; Poppi, R. J.; J. Pharm. Biomed. Anal. 2004, 36, 743.

12. El-Obeid, H. A.; Al-Badr, A. A. Em Analytical Profiles of Drug Substances 14; Florey, K., ed.; Academic Press: San Diego, 1985, p. 551.

13. Higgins, J. D.; Gilmor, T. P.; Martelucci, S. A.; Bruce, R. D.; Brittain, H G. Em Analytical Profiles of Drug Substances and Excipients 28; Florey, K., ed.; Academic Press: San Diego, 1999, p. 282.

14. van Staden, J. F.; Tsanwani, M.; Talanta 2002, 58, 1095.

15. Ramos, M. L.; Tyson, J. F.; Curran, D. J.; Anal. Chim. Acta 1998, 364, 107.

16. Milch, G.; Szabo, E.; J. Pharm. Biomed. Anal. 1991, 9, 1107.

17. Moreira, A. B.; Oliveira, H. P. M.; Atvars, T. D. Z.; Dias, I. L. T.; Neto, G. O.; Zagatto, E. A. G.; Kubota, L. T.; Anal. Chim. Acta 2005, 539, 257

18. Franeta, J. T.; Agbaba, D. D.; Eric, S. M.; Pavkov, S. P.; Vladimirov, S. D.; Alekisic, M. B.; J. Pharm. Biomed. Anal. 2001, 24, 1169.

19. Ramos-Martos, N.; Aguirre-Gomez, F.; Molina-Diaz, A.; Capitain-Vallvey, L. F.; J. AOAC Int. 2001, 84, 676.
20. Vieira, I. C.; Lupetti, K. O.; Fatibello, O.; Quim. Nova 2003, 26, 39.

21. Elragehy, N. A.; Abdelkawy, M.; Elbayoumy, A.; Anal. Lett. 1994, 27, 2127.

22. Hergert, L. A.; Escandar, G. M.; Talanta 2003, 60, 235.

23. Gasco-Lopez, A. L.; Izquierdo-Hornillos, R.; Jimenez, A.; J. Pharm. Biomed. Anal. 1999, 21, 143.

24. Tsao, J. C.; Savage, T. S.; Drug Develop. Ind. Pharm. 1985, 11, 1123.

25. Crowther, J. B.; Covery, T. R.; Deway, E. A.; Henion, J. D.; Anal. Chem. 1984, 56, 2921.

26. Lampert, B. M.; Stewart, J. T.; J. Chromatogr. 1990, 504, 381.

27. Donato, M. G.; Baeyens, W.; Vandenbossche, W.; Sandra, P.; J. Pharm Biomed. Anal. 1994, 12, 21.

28. Sadecka, J.; Cakrt, M.; Hercegova, A.; Polonski, J.; Skacani, I.; J. Pharm. Biomed. Anal. 2001, 25, 881.

29. Husain, S.; Kifayatullah, M.; Sekhar, R.; J. AOAC Int. 1994, 77, 1443.

30. Basu, D.; Mahalanabis, K. K.; Roy, B.; J. Pharm. Biomed. Anal. 1998, 16, 809 .

31. Barros Neto, B.; Scarminio, I. S.; Bruns, R. E.; Como Fazer Experimentos, Ed. da UNICAMP: Campinas, 2003, p. 91.

32. Hussain, S.; Murty, A. S. R.; Narasimha, R.; Indian Drugs 1989, 26, 557.

33. Takacs-Novak, K.; Box, K. J.; Avdeef, A.; Int. J. Pharm. 1997, 151, 235.

34. Tam, K. Y.; Takacs-Novak, K.; Anal. Chim. Acta 2001, 434, 157.

35. International Conference on Harmonization Tripartite Guideline Validation of Analytical Procedures: Methodology; Fed. Regist. 1997, vol. 62 , p. 27463

36. Agência Nacional de Vigilância Sanitária; Guia para Validação de Métodos Analíticos e Bioanalíticos, Resolução - RE no 899, de 29 de maio de 2003.

37. Lorber, A.; Faber, K.; Kowalski, B. R.; Anal. Chem. 1997, 69, 1620.

38. Booksh, K. S.; Kowalski, B. R.; Anal. Chem. 1994, 66, 782A

39. Cuadros-Rodriguez, L.; García-Campaña, A. M.; Jimenez-Linares, C.; Román-Ceba, M.; Anal. Lett. 1993, 26, 1243. 